\title{
Molecular Architecture Directs Linear-Bottlebrush-Linear Triblock Copolymers to Self-Assemble to Soft Reprocessable Elastomers
}

Shifeng Nian, ${ }^{1}$ Huada Lian, ${ }^{2}$ Zihao Gong, ${ }^{1}$ Mikhail Zhernenkov, ${ }^{3}$ Jian Qin,,${ }^{2,}$ Li-Heng Cai ${ }^{1,4,5, *}$

${ }^{1}$ Department of Materials Science and Engineering, ${ }^{4}$ Department of Chemical Engineering, ${ }^{5}$ Department of Biomedical Engineering, University of Virginia, Charlottesville, VA 22904, USA.

${ }^{2}$ Department of Chemical Engineering, Stanford University, Stanford, CA 94305, USA.

${ }^{3}$ National Synchrotron Light Source-II, Brookhaven National Laboratory, Upton, NY 11973, USA

${ }^{*}$ Correspondence and requests for materials should be addressed to L.-H. Cai (liheng.cai@virginia.edu) or J. Qin (jianq@stanford.edu).

\section{Supporting Information:}

SI Materials and Methods

SI Text

Figures S1-S16

Table S1-S4 


\section{$\underline{\text { SI Materials and Methods }}$}

\section{S1. Materials}

Monomethacryloxypropyl-terminated poly(dimethylsiloxane) (MCR-M07, average molar mass $800 \mathrm{~g} / \mathrm{mol}$; MCR-M11, average molar mass 1000 g/mol; MCR-M17, average molar mass 5000 g/mol; MCR-M22, average molar mass $10000 \mathrm{~g} / \mathrm{mol}$ ) were purchased from Gelest and purified using basic alumina columns to remove inhibitors. Copper(II) chloride $\left(\mathrm{CuCl}_{2}, 99.999 \%\right)$, tris[2-(dimethylamino)ethyl]amine $\left(\mathrm{Me}_{6} \mathrm{TREN}\right)$, ethylene bis(2-bromoisobutyrate (2-BiB, 97\%), Tin(II) 2-ethylhexanoate (Sn(EH)2, 92.5 $100 \%$ ), anisole $(\geq 99.7 \%)$ and xylene ( $\geq 99.7 \%)$ were purchased from Sigma Aldrich and used as received. Tetrahydrofuran (THF, analytical reagent (AR)), purchased from Macron Fine Chemicals, and THF (HPLC), purchased from Fisher, were used as received.

\section{S2. Synthesis and characterization of linear-bottlebrush-linear (LBBL) triblock copolymers}

We use activator regenerated by electron transfer (ARGET) atom transfer radical polymerization (ATRP) to synthesize the LBBL triblock copolymers. Here, we use one sample as an example to illustrate the details for the synthesis and characterization of LBBL polymers.

\section{S2.1. Bottlebrush polydimethylsiloxane}
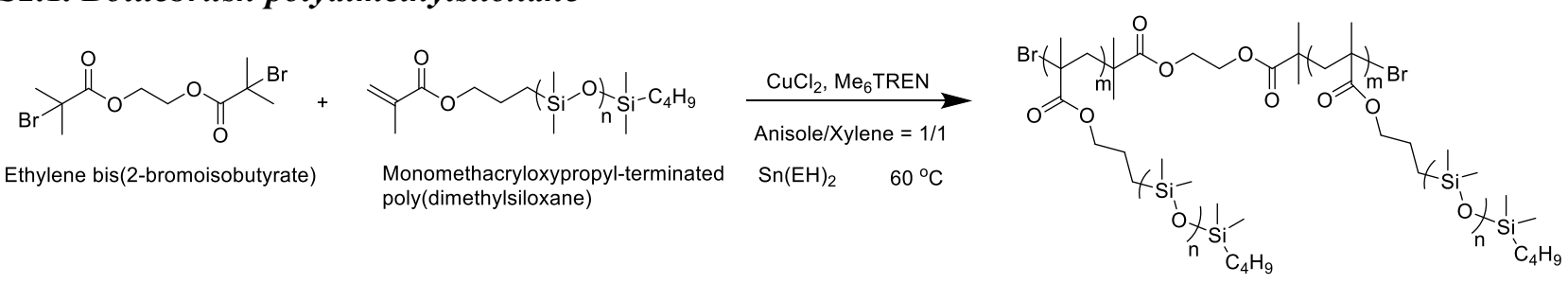

Figure S1. Synthetic of bottlebrush poly(dimethylsiloxane) (bbPDMS) polymer

We start by synthesizing the middle bottlebrush block, and the experimental conditions for bottlebrush poly(dimethylsiloxane) (PDMS) made of 500 side chains of $1 \mathrm{kDa}$ each is shown in Fig. S1. A $50 \mathrm{~mL}$ Schlenk flask is charged with ethylene bis(2-bromoisobutyrate) (2f-BiB, $3.6 \mathrm{mg}, 0.01 \mathrm{mmol}$ ), MCR-M11 $(8 \mathrm{~g}, 8 \mathrm{mmol})$, xylene $(4 \mathrm{~mL})$ and anisole $(4 \mathrm{~mL})$. Me ${ }_{6}$ TREN (46 mg, $\left.0.2 \mathrm{mmol}\right)$ and $\mathrm{CuCl}_{2}(2.7 \mathrm{mg}, 0.02$ $\mathrm{mmol}$ ) are dissolved in $1 \mathrm{~mL}$ DMF to make a catalyst solution. Then, $100 \mu \mathrm{L}$ catalyst solution, containing $0.02 \mathrm{mmol} \mathrm{Me}_{6}$ TREN and $0.002 \mathrm{mmol} \mathrm{CuCl}_{2}$, is added to the mixture. The mixture is bubbled with nitrogen for $60 \mathrm{~min}$ and then degassed by three freeze-pump-thaw cycles. During the final cycle, the flask is filled with nitrogen, and $\mathrm{Sn}(\mathrm{EH})_{2}(32 \mathrm{mg}, 0.08 \mathrm{mmol})$ in $300 \mu \mathrm{L}$ xylene is quickly injected to the reaction mixture using a syringe. The flask is sealed, evacuated, and backfilled with nitrogen five times and then immersed in an oil bath at $60{ }^{\circ} \mathrm{C}$. The conversion of the reaction is monitored by proton nuclear magnetic resonance spectroscopy $\left({ }^{1} \mathrm{H}\right.$ NMR). The reaction is stopped at about $60 \%$ conversion, which is confirmed by NMR. At such a conversion, the degree of polymerization 480, resulting a bbPDMS of 480 side chains of $1 \mathrm{kDa}$. The reaction mixture is then diluted with THF and passed through a neutral alumina column to remove the catalyst. The collected solution is concentrated using a rotary evaporator (Buchi R205). The concentrated polymer solution is precipitated in methanol for three times to remove unreacted monomers and other impurities. At room temperature, the purified bottlebrush polymer forms a viscous, transparent liquid.

${ }^{1} \mathrm{H}$ NMR analysis. NMR characterization is performed using Varian-600 MHz spectrometer. Chemical shifts for ${ }^{1} \mathrm{H}$ NMR spectra are reported in parts per million (PPM) relative to a singlet at $7.26 \mathrm{ppm}$ in $\mathrm{CDCl}_{3}$. An example of NMR spectra of purified bottlebrush PDMS is shown in Fig. $\mathbf{S 2}$. 


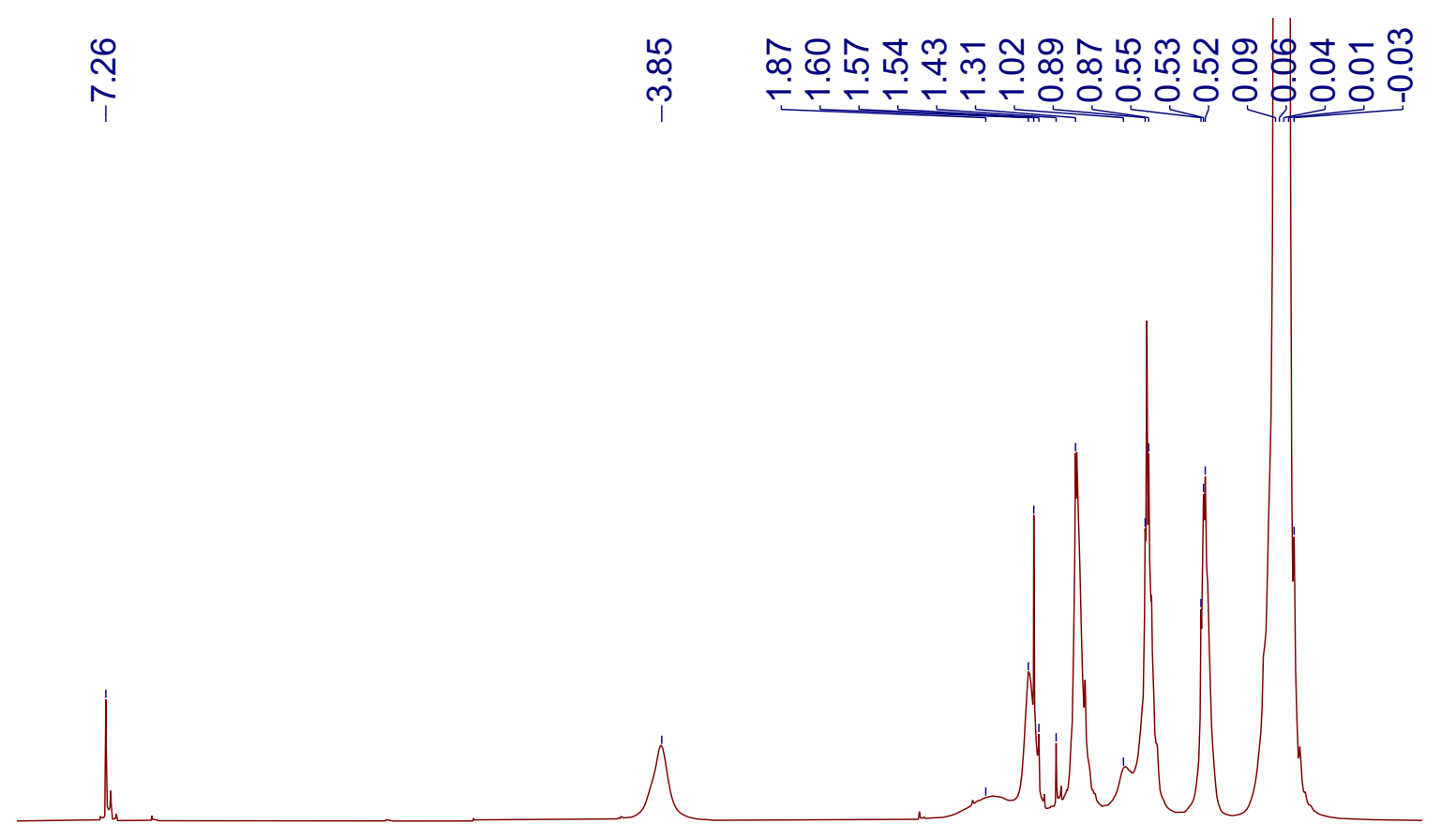

\section{$\begin{array}{lllllllllllllllll}7.5 & 7.0 & 6.5 & 6.0 & 5.5 & 5.0 & 4.5 & 4.0 & 3.5 & 3.0 & 2.5 & 2.0 & 1.5 & 1.0 & 0.5 & 0.0 & -0.5\end{array}$ \\ f1 (ppm)}

Figure S2. ${ }^{1} \mathrm{H}$ NMR data of bbPDMS with side chain MW oflkDa and DP 480. ${ }^{1} \mathrm{H}$ NMR (600 MHz, $\left.\mathrm{CDCl}_{3}\right) \delta(\mathrm{ppm}) 3.85\left(\mathrm{~m},-\mathrm{CO}-\mathrm{O}-\mathrm{CH}_{2}-\mathrm{CH}_{2}-\mathrm{CH}_{2}-\mathrm{Si}\left(\mathrm{CH}_{3}\right)_{2}-\mathrm{O}-\right), 1.87$ and $1.02\left(\mathrm{~m},-\mathrm{CH}_{2}-\mathrm{C}_{\left(\mathrm{CH}_{3}\right)\left(\mathrm{CH}_{2}-\right)-}\right.$ CO-O-), 1.60-1.54 (m, -CO-O- $\left.\mathrm{CH}_{2}-\mathrm{CH}_{2}-\mathrm{CH}_{2}-\mathrm{Si}\left(\mathrm{CH}_{3}\right)_{2}-\mathrm{O}-\right), 1.43$ (m, $\left.-\mathrm{CH}_{2}-\mathrm{C}\left(\mathrm{CH}_{3}\right)\left(\mathrm{CH}_{2}-\right)-\mathrm{CO}-\mathrm{O}-\right), 1.31$ (m, -O-Si $\left.\left(\mathrm{CH}_{3}\right)_{2}-\mathrm{CH}_{2}-\mathrm{CH}_{2}-\mathrm{CH}_{2}-\mathrm{CH}_{3}\right), 0.87$ (-O-Si $\left.\left(\mathrm{CH}_{3}\right)_{2}-\mathrm{CH}_{2}-\mathrm{CH}_{2}-\mathrm{CH}_{2}-\mathrm{CH}_{3}\right), 0.55-0.52$ (m, - $\mathrm{CH}_{2}-(-$ $\left.\left.\mathrm{Si}\left(\mathrm{CH}_{3}\right)_{2}-\mathrm{O}-\right)_{\mathrm{n}}-\mathrm{Si}\left(\mathrm{CH}_{3}\right)_{2}-\mathrm{CH}_{2}-\mathrm{CH}_{2}-\mathrm{CH}_{2}-\mathrm{CH}_{3}\right), \quad 0.09--0.03$ (m, - $\mathrm{CH}_{2}-\left(-\mathrm{Si}\left(\mathrm{CH}_{3}\right)_{2}-\mathrm{O}-\right)_{\mathrm{n}}-\mathrm{Si}\left(\mathrm{CH}_{3}\right)_{2}-\mathrm{CH}_{2}-\mathrm{CH}_{2}-$ $\left.\mathrm{CH}_{2}-\mathrm{CH}_{3}\right)$.

The number of side chains per bottlebrush is calculated based on the conversion of PDMS macromonomers to bottlebrush PDMS, which is measured by the NMR spectra of the raw reaction mixture (Fig. S3). For example, in Fig. S3, area a corresponds to the two H atoms on the carbon adjacent to the ester group in the PDMS macromonomer. Area a' corresponds to the two H atoms on the carbon adjacent to the ester group in the bbPDMS. Area $\mathbf{b}$ corresponds to the four $\mathrm{H}$ atoms on the two carbon atoms adjacent to the two end silicon atoms, either in PDMS macromonomer or bbPDMS. Area(a') + $\operatorname{Area}(\mathbf{a})=\operatorname{Area}(\mathbf{b}) / 2$. Therefore, for this synthesis:

$$
\text { Conversion }=\operatorname{Area}\left(\mathbf{a}^{\prime}\right) \times 100 \% /\left(\operatorname{Area}(\mathbf{a})+\operatorname{Area}\left(\mathbf{a}^{\prime}\right)\right)=(1-\operatorname{Area}(\mathbf{a}) \times 2 / \operatorname{Area}(\mathbf{b})) \times 100 \%
$$

In Fig. S3, the conversion of monomer $=(1-0.8 \times 2 / 4) \times 100 \%=60 \%$. The number of side chains $=[\mathrm{M}]$ $/[\mathrm{I}] \times$ conversion $=800 \times 0.6=480$ 


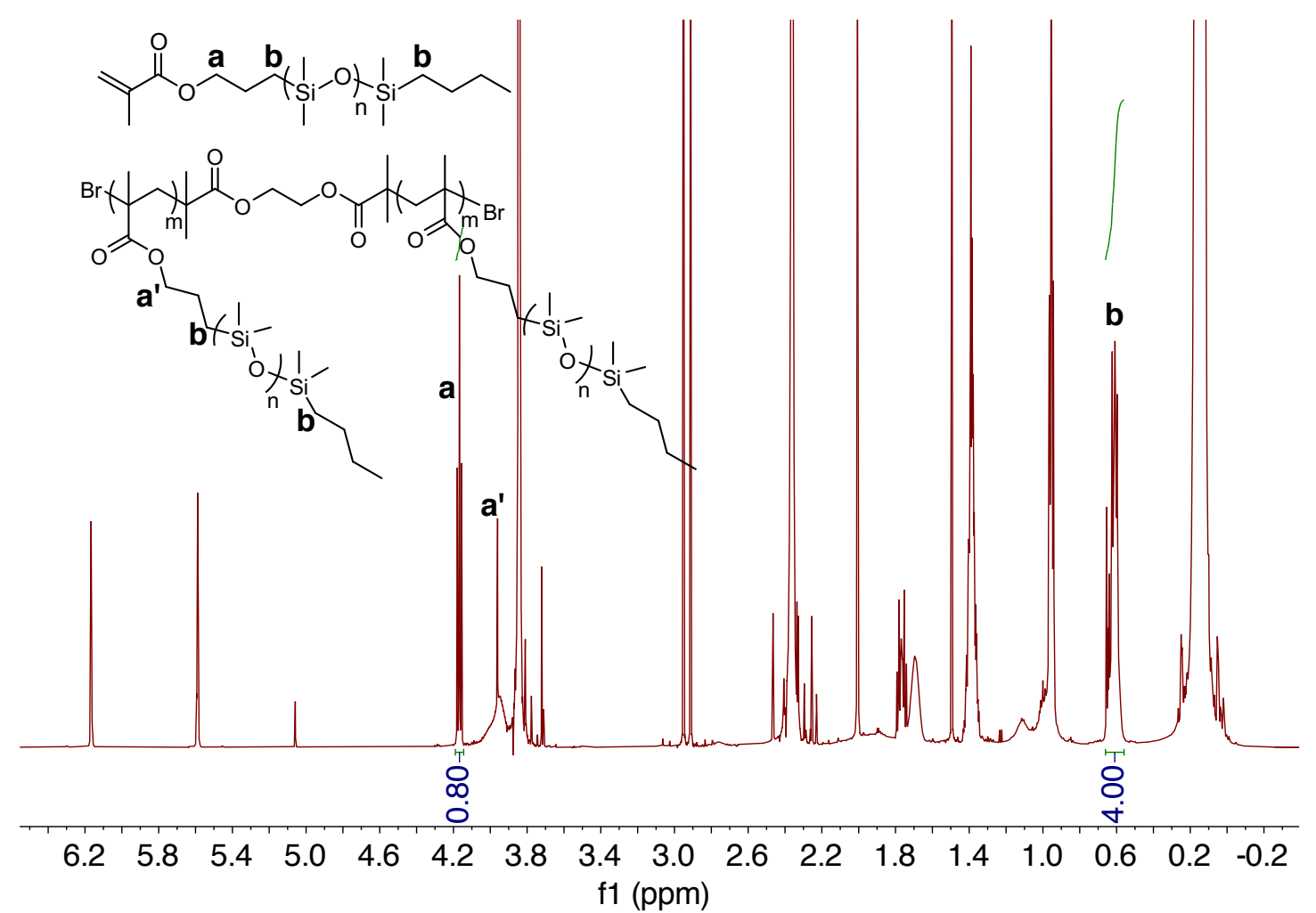

Figure S3. ${ }^{1} \mathrm{H}$ NMR data of raw mixture of ARGET ATRP of PDMS macromonomer. Conversion $=(1$ Area(a) $\times 2 /$ Area (b) $) \times 100 \%=60 \%$. The polymer synthesized is bbPDMS with side chain $1 \mathrm{kDa}$ and DP 480.

Gel permeation chromatography (GPC) characterization. The polydispersity index (PDI) of the polymer is characterized using GPC. GPC measurements are performed using TOSOH EcoSEC HLC-8320GPC system with two TOSOH Bioscience TSKgel GMH $\mathrm{HR}_{\mathrm{R}} \mathrm{M} 5 \mu \mathrm{m}$ columns in series and a refractive index detector at $40^{\circ} \mathrm{C}$. HPLC grade THF is used as the eluent with a flow rate of $1 \mathrm{~mL} / \mathrm{min}$. The calibration curve is obtained using standard polystyrene (PS) sample. The samples are dissolved in THF with a concentration around $3 \mathrm{mg} / \mathrm{mL}$. The GPC plot of this polymer is shown in Fig. S4. The PDI of the polymer is calculated using EcoSEC analysis software and its PDI is 1.31.

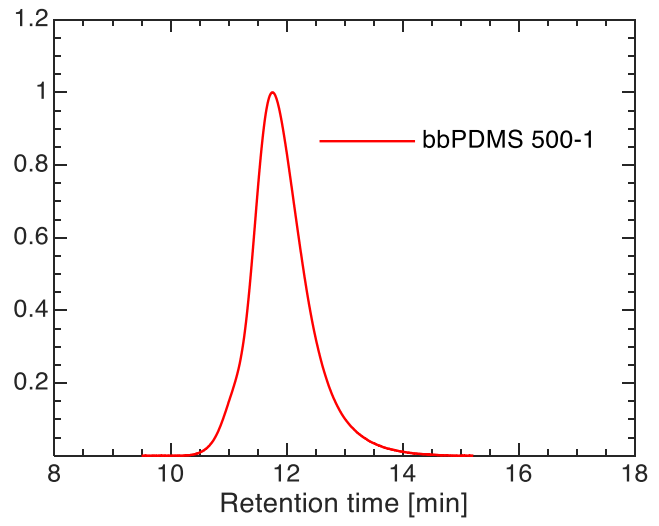

Figure S4. GPC data of bbPDMS with side chain $1 \mathrm{kDa}$ and degree of polymerization (DP) 480 . The PDI is 1.31. In the figure legend, 500 means the MW of bbPDMS is around $500 \mathrm{kDa}$, and 1 means the MW of PDMS side chain is $1 \mathrm{kDa}$. 
S2.2. Linear-bottlebrush-linear (LBBL) triblock copolymer

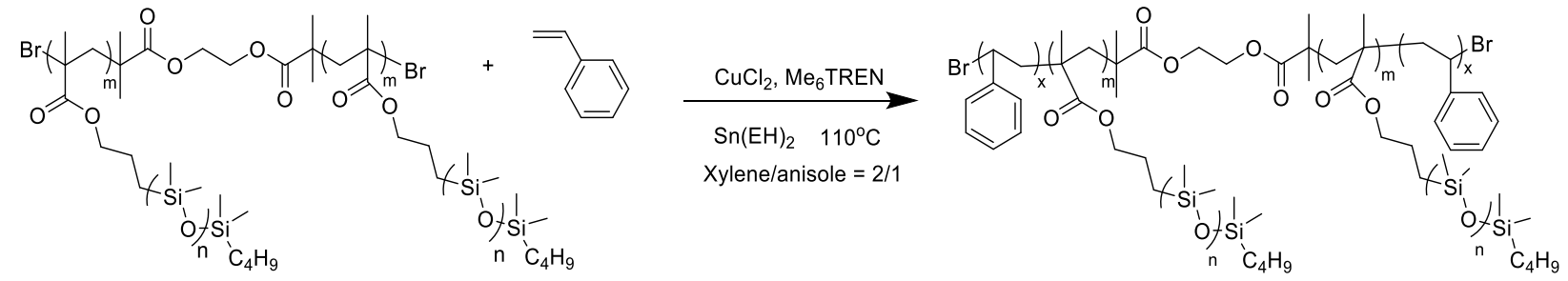

Figure S5. Synthetic route of bottlebrush poly(dimethylsiloxane) (PDMS) polymer

We use the bbPDMS obtained in $\mathbf{S 2 . 1}$ as a macroinitiator to synthesize a LBBL triblock polymer, as illustrated in Fig. S5. A $50 \mathrm{~mL}$ Schlenk flask is charged with styrene (275 mg, $2.64 \mathrm{mmol})$, macroinitiator (polyM11, 480kDa, $1.1 \mathrm{~g}, 0.0023 \mathrm{mmol})$ and anisole/xylene (v/v = 1/2) $(5 \mathrm{~mL}) . \mathrm{Me}_{6}$ TREN (46 mg, 0.2 $\mathrm{mmol})$ and $\mathrm{CuCl}_{2}(2.7 \mathrm{mg}, 0.02 \mathrm{mmol})$ are dissolved in $1 \mathrm{~mL}$ DMF to make a catalyst solution. Then, 22 $\mu \mathrm{L}$ catalyst solution (contains $0.0044 \mathrm{mmol} \mathrm{Me}{ }_{6} \mathrm{TREN}$ and $0.00044 \mathrm{mmol} \mathrm{CuCl}_{2}$ ) is added to the mixture. The solution is bubbled with nitrogen for $60 \mathrm{~min}$ and then degassed by three freeze-pump-thaw cycles. During the final cycle, the flask is filled with nitrogen, and $\mathrm{Sn}(\mathrm{EH})_{2}(7.2 \mathrm{mg}, 0.0176 \mathrm{mmol})$ is quickly added to the reaction mixture. The flask is sealed, evacuated, and backfilled with nitrogen five times and then immersed in an oil bath at $120{ }^{\circ} \mathrm{C}$ for $12 \mathrm{~h}$. The reaction mixture is diluted with THF and passed through a neutral alumina column to remove the catalyst, and the collected solution is concentrated by rotary evaporator. The polymer is precipitated in acetone. Then the polymer is re-dissolved in THF and precipitated in acetone for another two times to remove the monomer. A small amount of polymer is taken out for NMR analysis and GPC analysis. From NMR, the weight fraction is $6 \%$, which indicates that the MW of PS is about $14 \mathrm{kDa}$ for each end block. The LBBL triblock copolymer is a transparent, elastic solid.

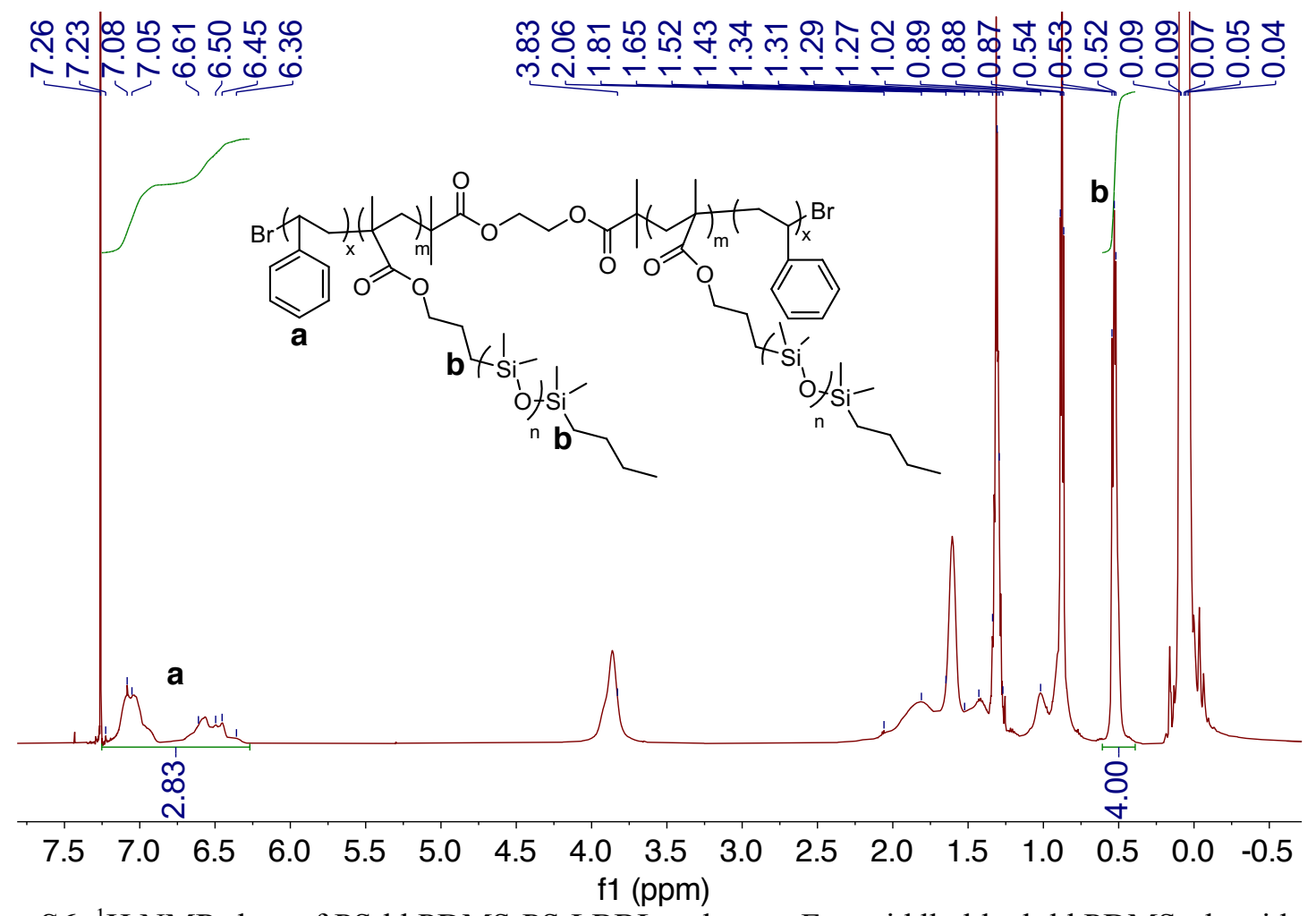

Figure S6. ${ }^{1} \mathrm{H}$ NMR data of PS-bbPDMS-PS LBBL polymer. For middle block bbPDMS, the side chain is $1 \mathrm{kDa}$ and degree of polymerization (DP) is 480 . The MW of PS for each end block is about $14 \mathrm{kDa}$. 
Weight fraction of total PS is around 6\%. ${ }^{1} \mathrm{H}$ NMR $\left(600 \mathrm{MHz}, \mathrm{CDCl}_{3}\right) \delta(\mathrm{ppm})$ 7.23-6.36 (m, -CH2$\mathrm{CH}(\mathrm{Ph})-\mathrm{CH} 2-, \mathrm{H}$ on phenyl ring), 3.83 (m, -CO-O- $\left.\mathrm{CH}_{2}-\mathrm{CH}_{2}-\mathrm{CH}_{2}-\mathrm{Si}\left(\mathrm{CH}_{3}\right)_{2}-\mathrm{O}-\right)$, 2.06-1.81, 1.43 and 1.02 $\left(\mathrm{m},-\mathrm{CH}_{2}-\mathrm{C}\left(\mathrm{CH}_{3}\right)\left(\mathrm{CH}_{2}-\right)-\mathrm{CO}-\mathrm{O}-\right.$ and $\left.-\mathrm{CH}_{2}-\mathrm{CH}(\mathrm{Ph})-\mathrm{CH}_{2}-\right)$ 1.65-1.52 (m, - $\mathrm{CO}-\mathrm{O}_{-}-\mathrm{CH}_{2}-\mathrm{CH}_{2}-\mathrm{CH}_{2}-\mathrm{Si}\left(\mathrm{CH}_{3}\right)_{2}-$ $\mathrm{O}-)$, 1.31-1.27 (m, -O-Si( $\left.\left.\mathrm{CH}_{3}\right)_{2}-\mathrm{CH}_{2}-\mathrm{CH}_{2}-\mathrm{CH}_{2}-\mathrm{CH}_{3}\right), 0.89-0.87$ (-O-Si( $\left.\left.\mathrm{CH}_{3}\right)_{2}-\mathrm{CH}_{2}-\mathrm{CH}_{2}-\mathrm{CH}_{2}-\mathrm{CH}_{3}\right), 0.54-$ 0.52 (m, $\left.-\mathrm{CH}_{2}-\left(-\mathrm{Si}\left(\mathrm{CH}_{3}\right)_{2}-\mathrm{O}-\right)_{\mathrm{n}}-\mathrm{Si}\left(\mathrm{CH}_{3}\right)_{2}-\mathrm{CH}_{2}-\mathrm{CH}_{2}-\mathrm{CH}_{2}-\mathrm{CH}_{3}\right), \quad 0.09-0.04 \quad\left(\mathrm{~m}, \quad-\mathrm{CH}_{2}-\left(-\mathrm{Si}\left(\mathrm{CH}_{3}\right)_{2}-\mathrm{O}-\right)_{\mathrm{n}}-\right.$ $\left.\mathrm{Si}\left(\mathrm{CH}_{3}\right)_{2}-\mathrm{CH}_{2}-\mathrm{CH}_{2}-\mathrm{CH}_{2}-\mathrm{CH}_{3}\right)$.

Determine the weight fraction of PS using NMR. We use NMR spectra to determine the weight fraction of PS, $f$. An example of the NMR spectra of a purified LBBL triblock copolymer is shown Fig. S6. Area $\mathbf{a}$ corresponds to the five $\mathrm{H}$ on the benzene ring of styrene repeating unit. Area $\mathbf{b}$ corresponds to four $\mathrm{H}$ on the two carbon atoms connected with the silicon atom.

$$
\mathrm{DP} \text { of PS }=[\text { Area }(\mathbf{a}) / 5] /[\text { Area }(\mathbf{b}) / 4] \times \mathrm{DP} \text { of bbPDS }
$$

$$
f=(\text { DP of PS } \times \text { MW of styrene }) /(\text { MW of bbPDMS }+ \text { DP of PS } \times \text { MW of styrene }) \times 100 \% \text {. }
$$

Therefore, for this synthesis:

$$
\begin{gathered}
\text { DP of PS }=(2.83 / 5) /(4 / 4) \times 480=272 \\
f=272 \times 104.15 \mathrm{~g} / \mathrm{mol} /(480 \mathrm{k} \mathrm{g} / \mathrm{mol}+272 \times 104.15 \mathrm{~g} / \mathrm{mol}) \times 100 \%=6 \% \\
\text { MW of PS for each end block }=272 \times 104.15 / 2 \mathrm{~g} / \mathrm{mol}=14 \mathrm{k} \mathrm{g} / \mathrm{mol}
\end{gathered}
$$

The PDI of the LBBL triblock copolymer is 1.44, calculated based on the GPC curve in Fig. S7.

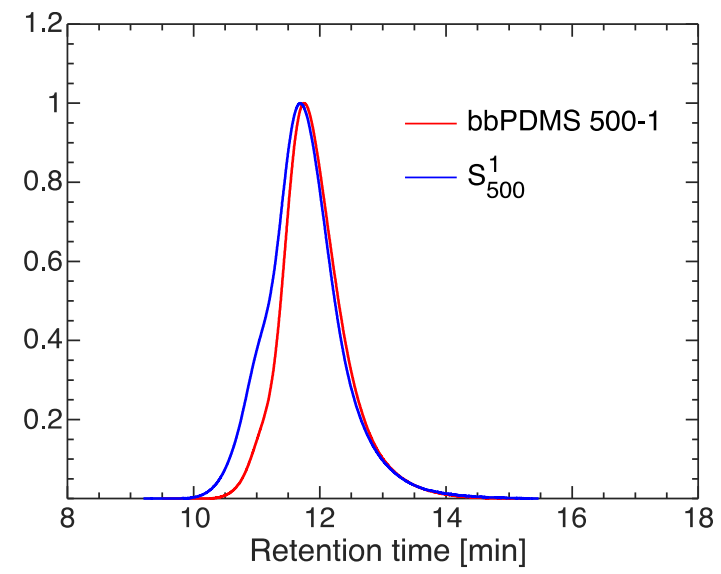

Figure S7. GPC characterization of bbPDMS and the corresponding PS- $b$-bbPDMS- $b$-PS LBBL polymer. For middle block bbPDMS, the side chain MW is $1 \mathrm{kDa}$ and DP is 480 . The MW of each PS block is $14 \mathrm{kDa}$. PDI of the LBBL polymer is 1.44 .

\section{S2.3. GPC and NMR characterization of all bbPDMS and LBBL triblock copolymers}

The GPC curves of six bbPDMS polymers and six LBBL polymers are shown in Figs. S8\&S9. The MW of each polymer is calculated using NMR instead of GPC. This is because the polystyrene standard for GPC calibration is linear, whereas the bbPDMS is of a branched architecture. For the same MW, a branched polymer has a smaller hydrodynamic radius, and therefore, would have a longer retention time. Consequently, the MW obtained using the calibration curve is not accurate, but the distribution of the MW or PDI remains valid. In our experiments, we use NMR to determine the number average MW of polymer samples, and use GPC to determine their PDI. The MW and PDI of all samples are summarized in Table S1. 


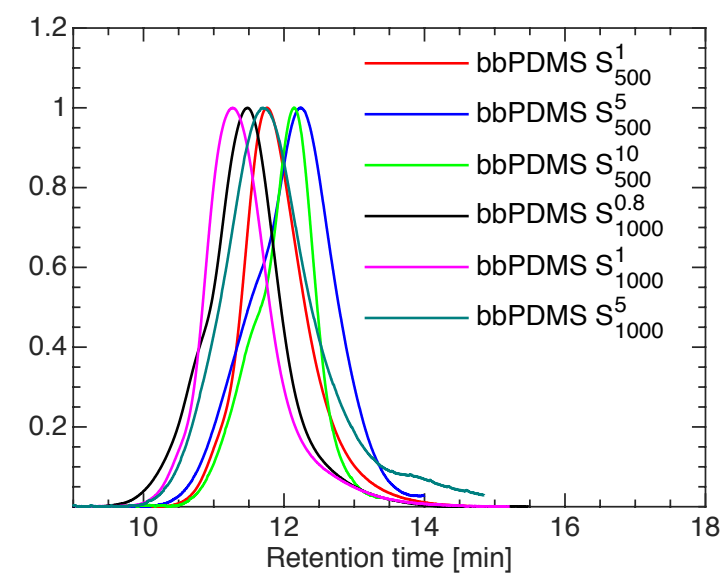

Figure S8. GPC data of six bbPDMS polymers.

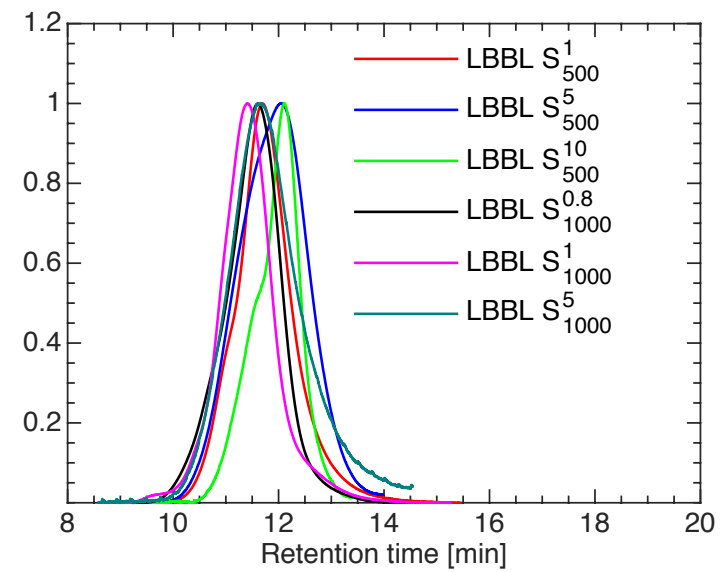

Figure S9. GPC data of six LBBL polymers.

Table S1. Molecular weight and PDI of bbPDMS and LBBL triblock copolymers

\begin{tabular}{|c|c|c|c|c|c|c|}
\hline \multicolumn{4}{|c|}{ bbPDMS polymer } & \multicolumn{3}{c|}{ LBBL triblock copolymer } \\
\hline Sample & $\begin{array}{c}\text { MW of } \\
\text { PDMS side } \\
\text { chains (kDa) }\end{array}$ & $\begin{array}{c}\text { MW of } \\
\text { bbPDMS } \\
(\mathrm{kDa})\end{array}$ & PDI & $\begin{array}{c}\text { MW of LBBL } \\
\text { copolymer } \\
(\mathrm{kDa})\end{array}$ & $\begin{array}{c}\text { Weight } \\
\text { fraction of PS } \\
f\end{array}$ & PDI \\
\hline$S_{500}^{1}$ & 1 & 480 & 1.31 & 508 & 0.06 & 1.44 \\
\hline$S_{500}^{5}$ & 5 & 520 & 1.46 & 550 & 0.06 & 1.55 \\
\hline$S_{500}^{10}$ & 10 & 470 & 1.44 & 490 & 0.04 & 1.51 \\
\hline$S_{1000}^{0.8}$ & 0.8 & 970 & 1.37 & 990 & 0.02 & 1.42 \\
\hline$S_{1000}^{1}$ & 1 & 1050 & 1.43 & 1070 & 0.02 & 1.46 \\
\hline$S_{1000}^{5}$ & 5 & 900 & 1.70 & 922 & 0.02 & 1.75 \\
\hline
\end{tabular}

\section{S3. Transmission Electron Microscopy (TEM) characterization of LBBL polymers}

Sample preparation. To ensure that the self-assembled microstructure is an equilibrium configuration, we use a combination of solvent and thermal annealing. For solvent annealing, we use toluene, a solvent equally good for PS and PDMS ${ }^{1,2}$, to eliminate possible effects of solvent selectivity on self-assembly ${ }^{3}$ and to ensure that the self-assembly approaches an equilibrium configuration as closely as possible. The 
solvent-annealed sample is then subject to thermal annealing at $120^{\circ} \mathrm{C}$, above the glass transition temperature $100^{\circ} \mathrm{C}$ of PS.

Specifically, a LBBL polymer sample is dissolved in toluene with a concentration of $1 \mathrm{mg} / \mathrm{mL}$. Then, $10 \mu \mathrm{L}$ polymer solution is added to a carbon film coated copper TEM grid. The sample is placed in fume hood at room temperature for $30 \mathrm{~min}$ to evaporate the solvent, and then is transferred to an oven filled with nitrogen for annealing at $120^{\circ} \mathrm{C}$ for $3 \mathrm{~h}$. After annealing, the oven is slowly cooled down to room temperature.

The same thermal annealing procedure is used to prepare samples for GISAXS measurements.

TEM imaging. TEM study was conducted on a FEI Titan TEM instrument. To characterize the microstructure self-assembled by LBBL polymers, we use a TEM (FEI Titan) operated at hollow-cone dark-field model with the electron energy of $300 \mathrm{keV}$. Example TEM images of LBBL samples are shown in Fig. S10.
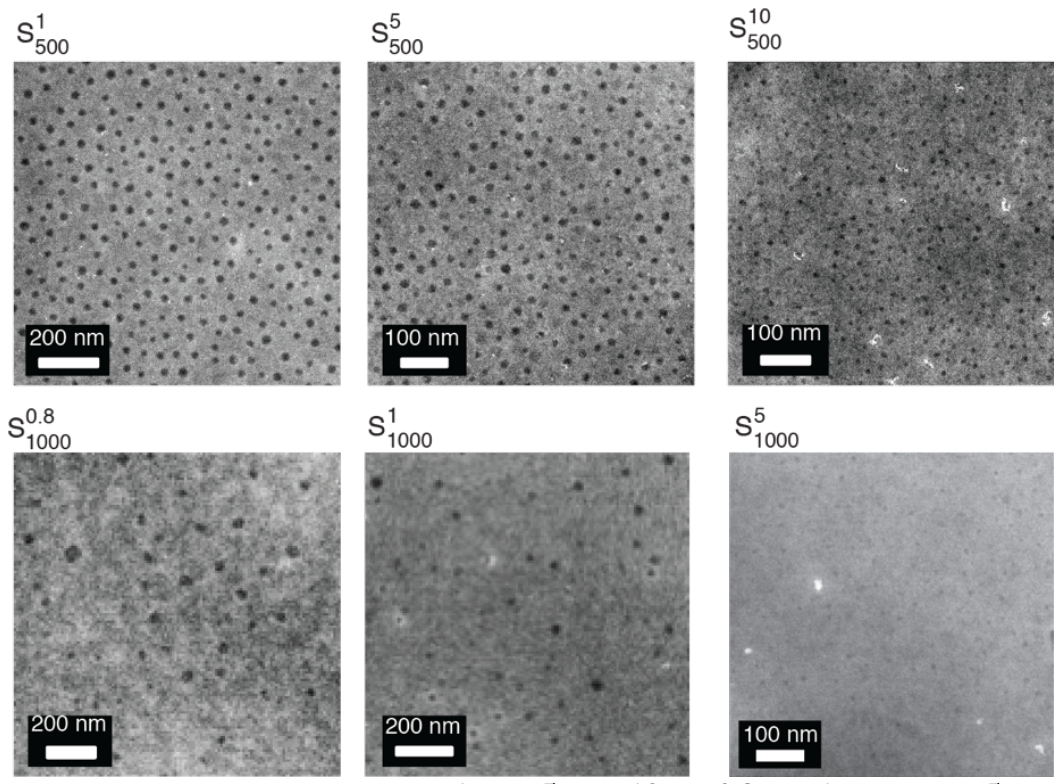

Figure S10. TEM images of LBBL polymers: $S_{500}^{1}, S_{500}^{5}, S_{500}^{10}, S_{1000}^{0.8}, S_{1000}^{1}$, and $S_{1000}^{5}$

Data analysis. We use ImageJ to calculate the PS domain radius and the distance between the centers of neighboring PS domains. The histograms of domain distance, $d$, and domain radius, $r$, of six LBBL samples are shown in Figs. S11\&S12, and their values are summarized in Table S2. 

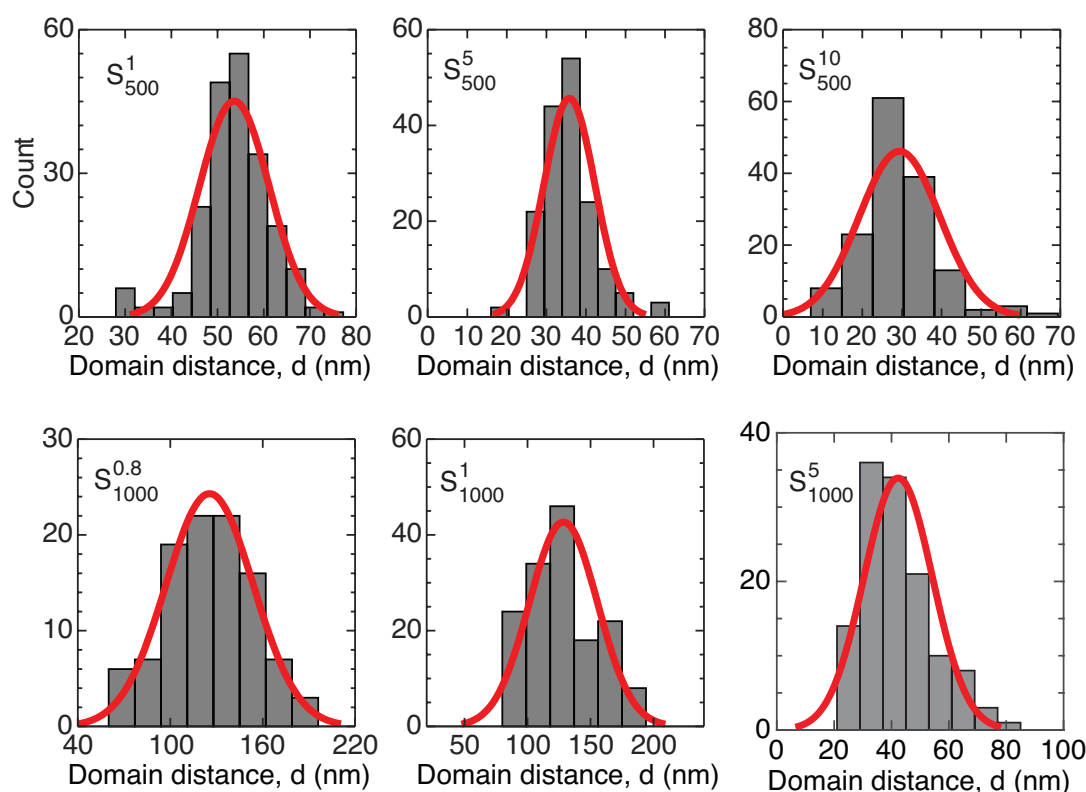

Figure S11. Histograms of domain distance of LBBL polymers: $S_{500}^{1}, S_{500}^{5}, S_{500}^{10}, S_{1000}^{0.8}, S_{1000}^{1}$, and $S_{1000}^{5}$.
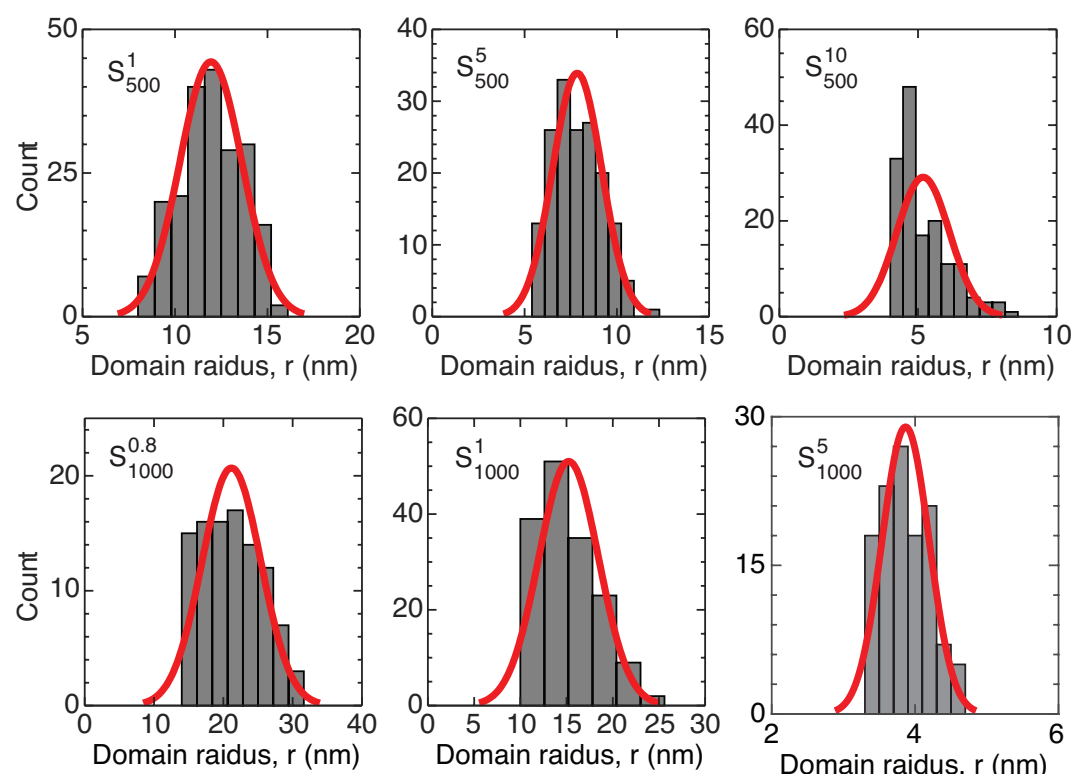

Figure S12. Histograms of domain radius of LBBL polymers: $S_{500}^{1}, S_{500}^{5}, S_{500}^{10}, S_{1000}^{0.8}, S_{1000}^{1}$, and $S_{1000}^{5}$.

Table S2. Domain distance, $d$, and radius, $r$, of LBBL polymers measured from TEM. Error represents standard deviation of $>100$ points.

\begin{tabular}{|c|c|c|c|c|c|c|}
\hline Sample & $S_{500}^{1}$ & $S_{500}^{5}$ & $S_{500}^{10}$ & $S_{1000}^{0.8}$ & $S_{1000}^{1}$ & $S_{1000}^{5}$ \\
\hline$d(\mathrm{~nm})$ & $53.6 \pm 7.5$ & $35.8 \pm 6.4$ & $29.0 \pm 10.2$ & $125.5 \pm 28.4$ & $128.4 \pm 27.0$ & $42.3 \pm 11.9$ \\
\hline$r(\mathrm{~nm})$ & $11.9 \pm 1.7$ & $7.8 \pm 1.3$ & $5.2 \pm 0.9$ & $21.2 \pm 4.2$ & $15.2 \pm 3.2$ & $3.8 \pm 0.3$ \\
\hline
\end{tabular}




\section{S4. Grazing-incidence small-angle scattering (GISAXS) measurements}

Thin film preparation. Thin films are prepared using dynamic spin coating. For $S_{500}^{1}$ and $S_{500}^{5}$ samples, the LBBL polymers are dissolved in toluene with a concentration of $100 \mathrm{mg} / \mathrm{mL}$. For $S_{1000}^{0.8}$ and $S_{1000}^{1}$ samples, they are dissolved in toluene with concentration of $80 \mathrm{mg} / \mathrm{mL}$. Then, a $1 \mathrm{~cm} \times 1 \mathrm{~cm}$ silicon wafer is loaded on the spin coater. The spin rate per minute (RPM) is set to 2000, and the spin time is 40s. For each solution, $30 \mu \mathrm{L}$ is taken out by a pipette and dropped on the silicon wafer after reaching a stable RPM. The thickness of the thin films can be controlled by casting the toluene solution of LBBL copolymers with different concentrations. The samples are then subject to temperature annealing following the same procedure as described for preparing TEM samples.

GISAXS measurements and data analysis. GISAXS measurements are carried out at the Soft Matter Interfaces (SMI, 12-ID) ${ }^{4}$ beamline at National Synchrotron Light Source-II in Brookhaven National Laboratory. The experiments are conducted using energy source of $16.1 \mathrm{keV}$ with sample-to-detector distance $7 \mathrm{~m}$ or energy source of $13.9 \mathrm{keV}$ with ample-to-detector distance $8.3 \mathrm{~m}$. The GISAXS patterns are recorded using a Pilatus $1 \mathrm{M}$ detector with a pixel size of $172 \mu \mathrm{m}^{2}$. An example of the scattered pattern is shown in Fig. S13 (left). We use GIXSGUI software ${ }^{5}$ to analyze the data, in which a region of interest (red rectangle in Fig. S13, left) is selected to calculate the dependence of intensity on the wavenumber, $q$, as shown in Fig. S13 (right). A characteristic peak is identified, and the domain distance is calculated using $d=2 \pi / q$. For example, for LBBL500-1 polymer, $q=0.0112 \AA^{-1}$, and $d=56 \mathrm{~nm}$. The error corresponds to the half-width of the peak based on fitting. The average domain distances for all samples are listed in Table S3.
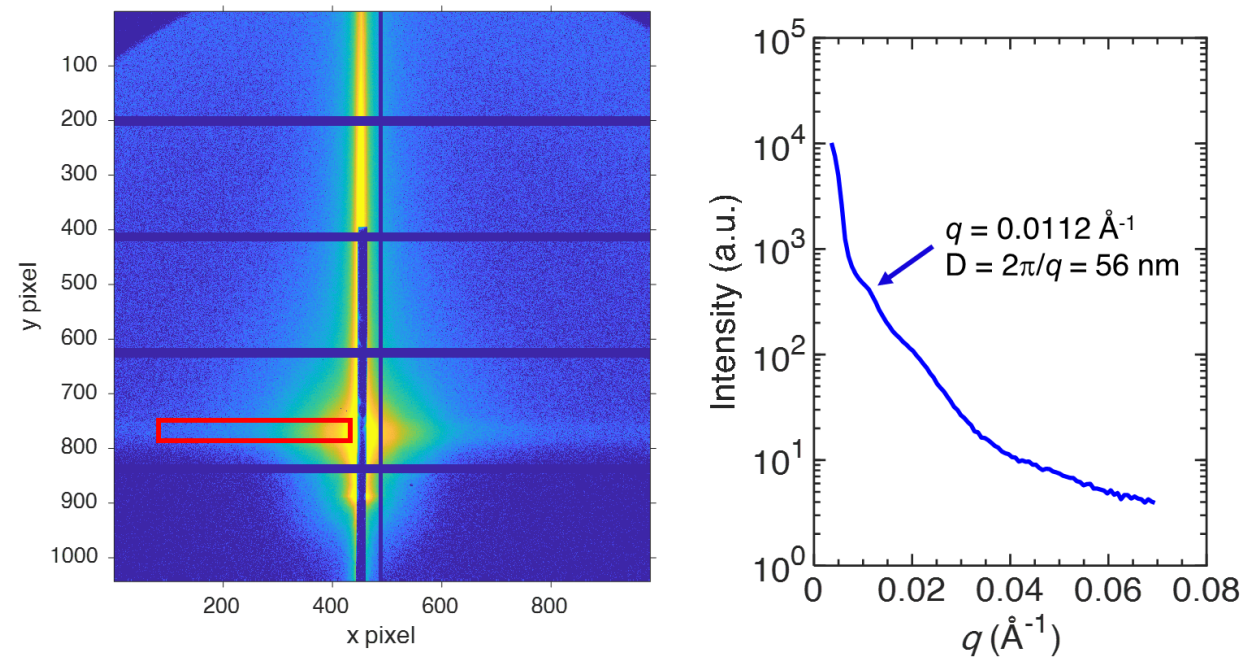

Figure S13. GISAXS pattern of sample $S_{500}^{1}$ (left) and the $q$ vs. intensity figure (right) after processing using GIXSGUI software.

Table S3. Domain distance of four LBBL polymers measured from GISAXS. The error bar represents half width of the peak from fitting.

\begin{tabular}{|c|c|c|c|c|}
\hline Sample & $S_{500}^{1}$ & $S_{500}^{5}$ & $S_{1000}^{0.8}$ & $S_{1000}^{1}$ \\
\hline$d(\mathrm{~nm})$ & $56.1 \pm 12.8$ & $32.2 \pm 10.3$ & $104.6 \pm 19.4$ & $110.1 \pm 21.5$ \\
\hline
\end{tabular}


S5. Rheology measurements are performed using a stress-controlled rheometer by a plate-plate geometry (Anton Paar MCR 320). In a typical measurement, we dissolve a LBBL polymer in THF to form a solution. The amount of solvent is adjusted carefully such that the solvent is just enough to fully dissolve the polymer. The solution is then transferred to the bottom plate of the rheometer. The temperature is set to $50^{\circ} \mathrm{C}$ for about $1 \mathrm{~h}$ to slowly evaporate the solvent. For frequency sweep, the shear strain is $0.5 \%$ and the temperature is $20^{\circ} \mathrm{C}$. For temperature sweep, the temperature range is from $0^{\circ} \mathrm{C}$ to $200^{\circ} \mathrm{C}$. The angular frequency is $0.1 \mathrm{rad} / \mathrm{s}$ and the shear strain is $0.5 \%$.

\section{S6. Reprocessability}

To demonstrate the reprocessability of the self-assembled elastomers, we re-dissolve an elastomer selfassembled by a LBBL triblock copolymer, $S_{500}^{5}$, using hexane, and re-dry the solution to obtain an elastomer. The reprocessed elastomer exhibits negligible changes in mechanical properties, as shown by the dynamic mechanical measurements in Fig. S14.

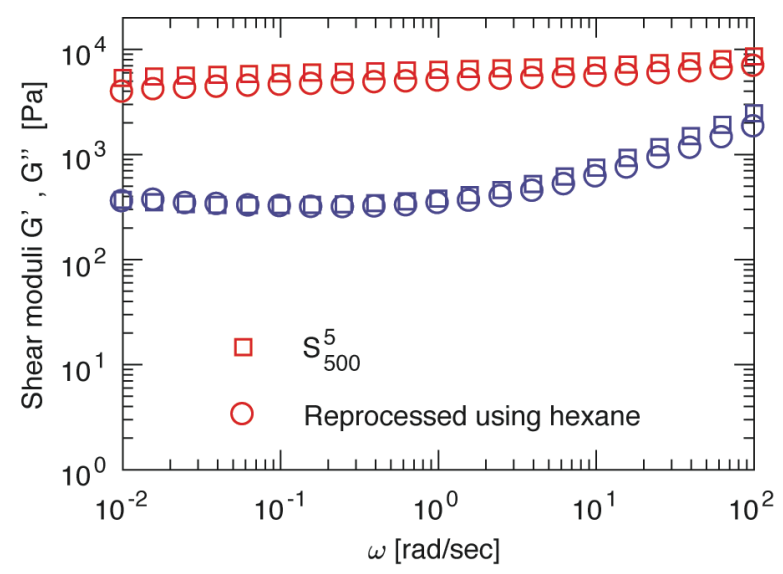

Figure S14. Elastomers self-assembled by a LBBL triblock copolymer show negligible changes in dynamic mechanical properties after being reprocessed using hexane. Measurements are performed at $20^{\circ} \mathrm{C}$

\section{S7. Modality analysis}

Some of the LBBL samples exhibit shoulder peaks on GPC profiles, suggesting a non-monomodal distribution of MW. Thus, one question is that whether the non-monomodal distribution of MW explains the large $(d-2 r) / L_{\max }$ and non-monotonic behavior of $(d-2 r) / R$ over the bottlebrush stiffness. $L_{\max }$ and $R$ are calculated based on the number of side chains per bottlebrush, which is determined based on NMR conversion rate rather than the GPC profile. The non-monomodal distribution of GPC profiles suggest samples are more polydisperse, which may result in wider distribution in measured characteristic lengths, but will not change the average values. Moreover, only samples with long side chains exhibit shoulder peaks. All GPC curves with shoulder peaks can be fitted by a bimodal distribution, and the weight fraction of minor component is less than $10 \%$ except for sample $S_{500}^{10}$, which has the longest side chain and the highest shoulder peak.

To illustrate the modality analysis, we use sample $S_{500}^{10}$ as an example. The retention profile of $S_{500}^{10}$ is well described by a bimodal distribution, one minor peak with a shorter retention time at $t_{s}=11.46 \mathrm{~min}$ and the other major peak with a longer retention time at $t_{l}=12.16 \mathrm{~min}$, as shown in Fig. S15. Since the area under a monomodal distribution denotes the total mass of the polymer, the weight fraction of the larger MW component is about $25 \%$. 


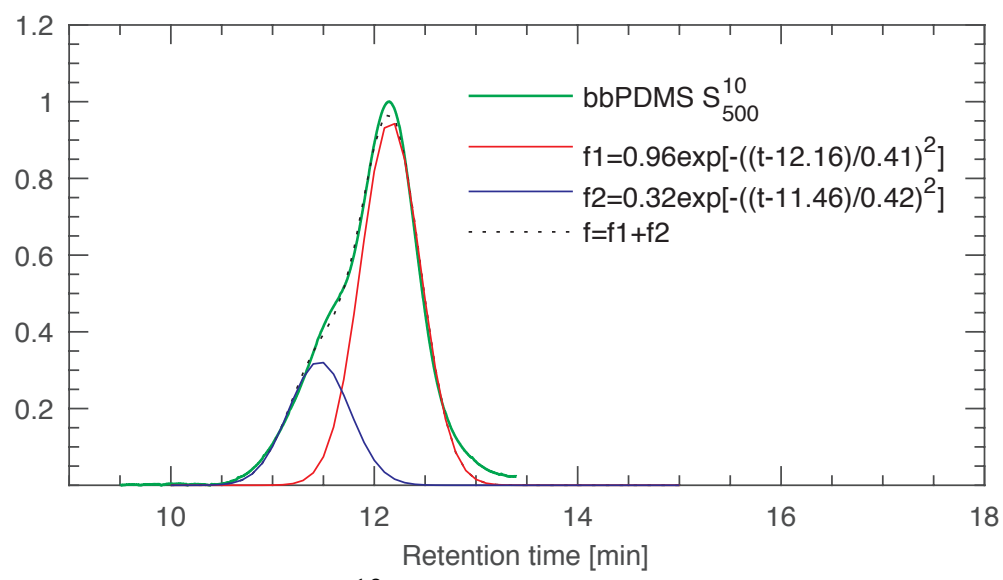

Figure S15. Modality analysis of sample $S_{500}^{10}$ reveals a bimodal distribution. The larger component (blue line) has a weight fraction of $25 \%$ and a number fraction of $\sim 10 \%$.

To convert the weight fraction to number fraction, one needs to determine the relation between MW ratio and the retention time ratio. For gel permeation chromatography, the elution volume, or time $t$, is linearly proportional to the logarithmic of MW (Appendix B.3.1 in ref. ${ }^{6}$ ). Therefore, the MW of a polymer is: $M=a \exp \left(-t / t_{0}\right)$, where parameters $a$ and $t_{0}$ are to be determined by column calibration. Because there are no GPC standards for bottlebrush polymers, we use our bottlebrush PDMS samples $S_{500}^{1}$ and $S_{1000}^{1}$ with no shoulder peaks as the standards for calibration. The average retention time for $S_{500}^{1}$ is $11.77 \mathrm{~min}$, and that for $S_{1000}^{1}$ is $11.27 \mathrm{~min}$. Considering the MW ratio is about 2 , it gives $t_{0} \approx 43 \mathrm{sec}$. Therefore, for the bimodal distribution of sample $S_{500}^{10}$, MW ratio between the two peaks is: $\frac{M_{t_{S}}}{M_{t_{l}}}=\exp \left(-\frac{t_{s}-t_{l}}{t_{0}}\right) \approx 2.7$. Note that the determined value $t_{0}$ is based on the samples with $1 \mathrm{kDa}$ side chains, which does not apply to samples with longer side chains, but this allows us to appreciate the MW ratio of the larger and smaller component in sample $S_{500}^{10}$. Nevertheless, the analysis suggests that the MW of the larger component is about twice of the smaller one. Therefore, the number fraction of larger MW polymer in the sample $S_{500}^{10}$ is about $10 \%$. As a result, the error of characteristic lengths attributed to non-monomodal distribution of polymers, if any, is less than $10 \%$ for all samples. This percentage is much less than the variation in the measured characteristic lengths.

Based on above discussion, we conclude that the non-monomodal distribution of LBBL polymers cannot explain the behavior of characteristic lengths and does not affect our conclusions in both microstructure and macroscopic mechanical properties. 


\section{SI Text}

Molecular parameters of bottlebrush polymers. The contour length $L_{\max }$, persistent length $l_{p}$, and end-to-end distance $R$ of a bottlebrush PDMS are calculated based on its molecular architecture. Consider a densely grafted bottlebrush formed by $n_{s c}$ side chains of $N_{s c}$ Kuhn monomers each. The contour length is independent of the side chain MW and is proportional to number of side chains,

$$
L_{\max }=n_{s c} l
$$

in which $l$ is the distance between two neighboring grafting sites along the backbone. In our experiments, the backbone of the bottlebrush PDMS is similar to poly(methyl methacrylate) (PMMA). Therefore, we use the size occupied by a chemical monomer in PMMA as the grafting distance. The Kuhn monomer size, $b$, of a PMMA is $1.53 \mathrm{~nm}$, and the mass is $M_{0}=598 \mathrm{~g} / \mathrm{mol}^{2}$. The mass of a methyl methacrylate chemical monomer is $m_{0}=100 \mathrm{~g} / \mathrm{mol}$. Therefore, in a Kuhn monomer, there are 6 chemical monomers, and each chemical monomer effectively has the size of $0.25 \mathrm{~nm}$. In our calculations, we use $l=0.25 \mathrm{~nm}$.

The persistence length, $l_{p}$, of a densely grafted bottlebrush is about its cross-section size, which was described by our previous work ${ }^{7}$ and others. ${ }^{8,9}$ Here we briefly summarize the physical arguments for calculating the cross-section size. For a bottlebrush, the side chains are densely grafted to a backbone polymer, occupying a cylindrical space surrounding the backbone. The cross-section size of the cylindrical space is about the size $R_{S c}$ of a side chain. Within such a cylindrical space, a side chain occupies a volume, $R_{s c}^{2} l$, that is the product of the cross-section area $R_{S c}^{2}$ and the distance between two neighboring grafting sites $l$. This volume is equal to the volume of a side chain itself, $N_{s c} v_{0}$, in which $v_{0}$ is the volume of a Kuhn monomer. Therefore, the cross-section size of the bottlebrush is $R_{s c} \approx$ $\left(N_{s c} v_{0} / l\right)^{1 / 2}$.

The persistence length of the bottlebrush polymer is about its cross-section size,

$$
l_{p} \approx R_{s c} \approx\left(N_{s c} v_{0} / l\right)^{1 / 2} \propto N_{s c}^{1 / 2}
$$

which increases with the side chain MW by a power of $1 / 2$.

The bottlebrush is essentially a 'fat' linear polymer. Therefore, we use worm-like chain model to calculate its end-to-end distance $R,{ }^{10}$

$$
R^{2}=2 l_{p} L_{\max }-2 l_{p}\left(1-\exp \left(-\frac{L_{\max }}{l_{p}}\right)\right)
$$

Above scaling arguments omit prefactors on the order of unity as confirmed by molecular dynamics simulations of bottlebrush molecules. ${ }^{8}$

For PDMS, the mass of a Kuhn monomer is $M_{0}=381 \mathrm{~g} / \mathrm{mol}$, the size is $b=1.3 \mathrm{~nm}$, and the volume is $v_{0}=6.50 \times 10^{-1} \mathrm{~nm}^{3}$. Using these numbers and eqs. (S1-S3), one obtains the contour length $L_{\text {max }}$, persistent length $l_{p}$, and end-to-end distance $R$ of a bottlebrush PDMS. Details for each sample are listed in Table S4.

Table S4. Calculated contour length $L_{\max }$, persistent length $l_{p}$, and end-to-end distance $R$ of a bottlebrush PDMS using eqs. (S1-S3)

\begin{tabular}{|c|c|c|c|c|c|c|}
\hline & $\begin{array}{c}M_{S c} \\
(\mathrm{kDa})\end{array}$ & $n_{s c}$ & $\begin{array}{c}L_{\max } \\
(\mathrm{nm})\end{array}$ & $l_{p}(\mathrm{~nm})$ & $\begin{array}{c}R \\
(\mathrm{~nm})\end{array}$ & $\frac{L_{\max }}{2 l_{p}}$ \\
\hline$S_{500}^{1}$ & 1 & 480 & 123 & 2.6 & 25.0 & 23.5 \\
\hline$S_{500}^{5}$ & 5 & 104 & 27 & 5.8 & 15.7 & 2.3 \\
\hline$S_{500}^{10}$ & 10 & 47 & 12 & 8.3 & 9.7 & 0.7 \\
\hline$S_{1000}^{0.8}$ & 0.8 & 1212 & 312 & 2.3 & 37.4 & 53.1 \\
\hline$S_{1000}^{1}$ & 1 & 1050 & 269 & 2.6 & 37.2 & 51.4 \\
\hline$S_{1000}^{5}$ & 5 & 180 & 46 & 5.8 & 21.6 & 4.0 \\
\hline
\end{tabular}


Dependence of domain radius on domain distance. In the self-assembled nanostructure, both the radius of spherical domains and the distance between two spherical domains increase with the decrease of side chain MW. To understand this behavior, we develop a scaling theory for the self-assembly of LBBL triblock copolymers. The self-assembly is driven by the minimization of free energy, which is primarily contributed from two parts: (1) interfacial energy between distinct domains and (2) chain stretching in the middle bottlebrush block. The contribution from the stretching of the end blocks is neglected as they are much smaller than that of the middle block as discussed in the end.

Let's consider a sphere microstructure self-assembled by a LBBL triblock copolymer. The weight fraction of end blocks is $f$, the radius of a sphere domain is $r$, and the distance between the centers of two neighboring spherical domains is $d$. Within a volume $V$, there are $n_{s} \approx V / d^{3}$ spherical domains. The total interfacial area is $A \approx 4 \pi n_{s} r^{2}$. Considering that the density of different blocks is nearly the same, $f \approx$ $\left(\frac{r}{d}\right)^{3}$, and therefore, the interfacial area is $A \approx V f / r$. The interfacial energy density is the product of interfacial tension $\gamma$ and the interfacial area normalized by volume,

$$
F_{\text {int }} \approx \gamma A / V \approx \gamma f / r
$$

which increases linearly with $f$ but is inverse to the radius of spherical domains.

Stretching of the middle bottlebrush block. The stretching free energy of the middle block treated as a worm-like chain can be analyzed in two limits. In the weak stretching limit, the free energy obeys the same form as Gaussian coil. One middle block with end-to-end distance $d-2 r$ has a free energy: $F_{\text {ent }}^{0} \approx$ $k_{B} T \frac{(d-2 r)^{2}}{R^{2}} \approx k_{B} T \frac{d^{2}}{R^{2}}\left(1-f^{\frac{1}{3}}\right)^{2} \approx k_{B} T \frac{r^{2}}{R^{2}} f^{-\frac{2}{3}}$ for $f \ll 1$, where $R$ is the end-to-end distance. Denote the volume of a single chain by $V_{c}$, the total entropic free energy density due to the stretching of the middle block is

$$
F_{\text {ent }} \approx \frac{k_{B} T}{V_{c}} f^{-\frac{2}{3}} \frac{r^{2}}{R^{2}}
$$

The term $R^{2}$ relates to the molecular weight through $R^{2}=2 l_{p} L_{\text {max }}$, and the chain volume can be written as $V_{c}=p R^{2}=2 p l_{p} L_{\text {max }}$, where $p$ is the packing length of the bottlebrush block. Therefore, eq. S5 can be written as

$$
F_{\text {ent }} \approx \frac{k_{B} T}{4 p l_{p}^{2}} f^{-\frac{2}{3}} \frac{r^{2}}{L_{\text {max }}^{2}}
$$

Using eqs. S4 and S5, the density of total free energy is

$$
\frac{F_{t o t}}{k_{B} T} \approx \frac{\gamma}{k_{B} T} \frac{f}{r}+\frac{f^{-2 / 3}}{4 p l_{p}^{2}} \frac{r^{2}}{L_{\max }^{2}}
$$

Minimizing the density of free energy by setting $\left.\frac{\partial}{\partial r}\left(\frac{F_{t o t}}{V k_{B} T}\right)\right|_{r}=0$ gives the optimal radius of the spherical domains in equilibrium:

$$
r^{*} \approx 2 h^{-2 / 3} f^{5 / 9} p^{1 / 3} l_{p}^{4 / 3} \kappa^{2 / 3}
$$

in which $\kappa=L_{\max } / l_{p}$ as defined in the text. Here $h \equiv\left(k_{B} T / \gamma\right)^{\frac{1}{2}}$ is related to the interfacial thickness, and has the scale of a monomer length. Using the room temperature interfacial tension between PS and $\operatorname{PDMS}^{11} \gamma \approx 5 \times 10^{-3} \frac{\mathrm{N}}{\mathrm{m}}, h$ is estimated to be $1 \mathrm{~nm}$. For a bottlebrush, the effective Kuhn monomer is nearly spherical and thus the packing length is comparable to the persistence length, $p \approx l_{p}$. Therefore,

$$
r^{*} \approx 2 h^{-2 / 3} f^{5 / 9} l_{p}^{5 / 3} \kappa^{2 / 3}
$$

This scaling is expected to hold for large $\kappa$ values and for chains under small tension. The prefactor of the scaling relation can be determined by the fitting experimental, as shown by the solid line in Fig. $\mathbf{3 b}$. The scaling relation (eq. S9) suggests that for a sphere phase, both the domain radius and distance increase with the end-to-end distance of bottlebrush polymers, which depends on both the stiffness and contour length (eq. S3). Our results highlight the importance to control the molecular architecture of the bottlebrush polymer to obtain nanostructure of large characteristic length scales, which are important to the development polymer photonic crystals. ${ }^{12-17}$ 
In the strong stretching limit, the end-to-end distance of the middle block approaches $L_{\max }$ the stretching free energy can be calculated as follows. The force-extension rule of the worm-like chain near the full extension is given by $R_{z}=L_{\max }\left(1-\sqrt{k_{B} T / 4 l_{p} \tau}\right)$, in which $\tau$ is the force applied to hold the chain ends at separation $R_{z} \cdot{ }^{18}$ Integrating this equation of state, in which $z=L_{\max }\left(1-\sqrt{k_{\mathrm{B}} T /\left(4 l_{\mathrm{p}}\right)} / \sqrt{\tau}\right)$ is the instantaneous separation, by starting from an arbitrary reference $R_{z, 0}$ gives the free energy

$$
F\left(R_{Z}\right)=-\int_{R_{z, 0}}^{R_{Z}} d z \tau=\frac{k_{B} T L_{\max }^{2}}{4 l_{p}\left(L_{\max }-R_{Z}\right)}+\text { const. }
$$

Notice that the divergence at the $R_{z}=L_{\max }$ restricts the full extension. Then the total free energy density in this limit is given by

$$
\frac{F_{\text {int }}+F_{\text {ent }}}{k_{B} T}=\frac{f}{h^{2} r}+\frac{1}{V_{c}} \frac{L_{\max }^{2}}{4 l_{p}\left(L_{\max }-f^{-1 / 3} r\right)}
$$

Here the chain volume again can be written $V_{c}=2 p l_{p} L_{\max }$. Minimizing the free energy by varying $r$ yields

$$
r^{*}=\frac{f^{1 / 3} L_{\max }}{1+h f^{-1 / 3} p^{-1 / 2} L_{\max }^{1 / 2} l_{p}^{-1}}
$$

The packing length is comparable to the persistence length as discussed above. The fully stretched limit is more readily reached when the ratio $L_{\max } / l_{p}$ approaches unity. Furthermore, $h$ as a microscopic parameter is much smaller than the persistence length of the bottlebrush block. Consequently, the denominator is essentially unity, and we have

$$
r^{*} \approx f^{1 / 3} L_{\max } \approx 2 l_{p} f^{1 / 3} \kappa
$$

The linear relation suggests that despite the sphere domains are formed by end linear blocks, the size of spheres is controlled by the contour length of the bottlebrush block rather than that of the end linear chains. However, our experimental data does not support this scaling, likely because this regime requires a fully extended bottlebrush and is very narrow.

Stretching of the end blocks. The stretching free energy of the end blocks is neglected in the above consideration. Here we show that including this term does not change the $2 / 3$ scaling in the weakly stretched regime, which was followed by our data. The total stretching free energy of the middle and the end blocks can be written

$$
F_{\text {ent }} \approx \frac{k_{B} T}{V_{c}}\left(\frac{r^{2}}{f^{2 / 3} R_{m}^{2}}+2 \frac{r^{2}}{R_{e}^{2}}\right)
$$

Here $R_{m}^{2}=V_{m} / p_{m}$ and $R_{e}^{2}=V_{e} / p_{e}$ are the mean squared end-to-end distances of the middle and the end blocks in the non-stretched coil state, $V_{m}$ and $V_{e}$ are the volumes of the corresponding blocks, and $p_{m}$ and $p_{e}$ are the packing lengths. The factor 2 accounts for the contributions from the two end blocks. The middle block volume $V_{m}$ can be approximated by the chain volume $V_{c}$. The end-block volume $V_{e}$ is smaller by a factor $f / 2$. Therefore, eq. S14 can re-written as:

$$
F_{\text {ent }} \approx k_{B} T\left(\frac{p_{m}}{f^{2 / 3}}+\frac{4 p_{e}}{f}\right) \frac{r^{2}}{V_{c}^{2}}
$$

Since $V_{c}$ is proportional to $L_{\max }$, the last fraction has a similar scaling form as eq. S6 and will lead to the same $2 / 3$ scaling. The correction from the end blocks is small because $p_{e}$ is much less than $p_{m}$. Thus, it is reasonable to neglect the contribution to the free energy from the stretching of end linear blocks.

Effects of packing-induced strain of side chains on sphere radius. The steric hindrance between overlapping, long side chains would affect the sphere radius. To avoid packing frustration, the side chains near the sphere surface can rearrange by stretching outwards from the center of the sphere, as illustrated in Fig. S16. ${ }^{19}$ The stretching of these side chains results in the loss of the number of configuration states and thus the gain of entropic free energy, which is expected to be balanced by the loss of interfacial free 
energy, or the increase of sphere radius. As a result, the packing-induced stretching of side chains is expected to contribute to an increase, not a decrease, of sphere radius.

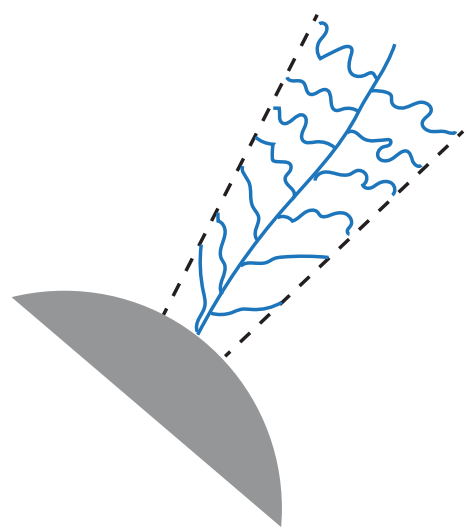

Figure S16. Illustration of packing-induced stretching of side chains near the sphere surface.

The packing-induced stretching occurs at a finite thickness away from the sphere surface, however; such a thickness is much smaller that the size of bottlebrush for most samples except for the stiffest sample, $S_{500}^{10}$. Therefore, we do not expect that the packing-induced stretching alters the conclusions obtained from scaling analysis.

The packing-induced strain becomes more pronounced for longer side chains, which would induce additional extension of bottlebrush backbone. For instance, for sample $S_{500}^{10}$ the size of side chains is slightly larger than the contour length in an unperturbed state. It is expected the nearly all side chains are subject to packing-induced strain; this may cause a strong tension that is large enough to stretch the C$\mathrm{C}$ bond along the backbone, resulting in a strongly stretched bottlebrush with size larger than the contour length. 


\section{References}

(1) Chu, J. H.; Rangarajan, P.; Adams, J. L. M.; Register, R. A. Morphologies of Strongly Segregated Polystyrene-Poly(Dimethylsiloxane) Diblock Copolymers. Polymer 1995, 36 (8), 1569-1575.

(2) Mark, J. E. Physical Properties of Polymers Handbook; Mark, J. E., Ed.; Springer Science \& Business Media, 2007.

(3) Kim, G.; Libera, M. Morphological Development in Solvent-Cast Polystyrene-PolybutadienePolystyrene (SBS) Triblock Copolymer Thin Films. Macromolecules 1998, 31 (8), 2569-2577.

(4) Zhernenkov, M.; Canestrari, N.; Chubar, O.; DiMasi, E. Soft Matter Interfaces Beamline at NSLSII: Geometrical Ray-Tracing vs. Wavefront Propagation Simulations. In Advances in Computational Methods for X-Ray Optics III; Sanchez del Rio, M., Chubar, O., Eds.; International Society for Optics and Photonics, 2014; Vol. 9209, p 92090G.

(5) Jiang, Z. GIXSGUI: A MATLAB Toolbox for Grazing-Incidence X-Ray Scattering Data Visualization and Reduction, and Indexing of Buried Three-Dimensional Periodic Nanostructured Films. J. Appl. Crystallogr. 2015, 48 (3), 917-926.

(6) Cai, L.-H. Structure and Function of Airway Surface Layer of the Human Lungs \& Mobility of Probe Particles in Complex Fluids, University of North Carolina at Chapel Hill, 2012.

(7) Cai, L. H.; Kodger, T. E.; Guerra, R. E.; Pegoraro, A. F.; Rubinstein, M.; Weitz, D. A. Soft Poly(Dimethylsiloxane) Elastomers from Architecture-Driven Entanglement Free Design. Adv. Mater. 2015, 27 (35), 5132-5140.

(8) Paturej, J. J.; Sheiko, S. S.; Panyukov, S.; Rubinstein, M. Molecular Structure of Bottlebrush Polymers in Melts. Sci. Adv. 2016, 2, e1601478.

(9) Daniel, W. F. M. M.; Burdyńska, J.; Vatankhah-Varnoosfaderani, M.; Matyjaszewski, K.; Paturej, J.; Rubinstein, M.; Dobrynin, A. V.; Sheiko, S. S. Solvent-Free, Supersoft and Superelastic Bottlebrush Melts and Networks. Nat. Mater. 2016, 15 (2), 183-189.

(10) Rubinstein, M.; Colby, R. H. Polymer Physics; Oxford University Press: Oxford ; New York, 2003.

(11) Biresaw, G.; Carriere, C. J.; Sammler, R. L. Effect of Temperature and Molecular Weight on the Interfacial Tension of PS/PDMS Blends. Rheol. Acta 2003, 42 (1), 142-147.

(12) Urbas, A. M.; Maldovan, M.; DeRege, P.; Thomas, E. L. Bicontinuous Cubic Block Copolymer Photonic Crystals. Adv. Mater. 2002, 14 (24), 1850-1853.

(13) Kang, Y.; Walish, J. J.; Gorishnyy, T.; Thomas, E. L. Broad-Wavelength-Range Chemically Tunable Block-Copolymer Photonic Gels. Nat. Mater. 2007, 6 (12), 957-960.

(14) Park, C.; Yoon, J.; Thomas, E. L. Enabling Nanotechnology with Self Assembled Block Copolymer Patterns. Polymer 2003, 44 (22), 6725-6760.

(15) Edrington, B. A. C.; Urbas, A. M.; Derege, P.; Chen, C. X.; Swager, T. M.; Hadjichristidis, N.; Xenidou, M.; Fetters, L. J.; Joannopoulos, J. D.; Fink, Y.; et al. Polymer-Based Photonic Crystals. Adv. Mater. 2001, 13 (6), 421-425.

(16) Prasad, P. N. Polymers for Photonics. Int. J. Nonlinear Opt. Phys. 1994, 3 (4), 531-541.

(17) Rumi, M.; Bunning, T. J. Polymers in Photonics: Controlling Information by Manipulating Light. J. Polym. Sci. Part B Polym. Phys. 2014, 52 (3), 157.

(18) Marko, J. F.; Siggia, E. D. Stretching DNA. Macromolecules 1995, 28 (26), 8759-8770. 
(19) Clair, C.; Lallam, A.; Rosenthal, M.; Sztucki, M.; Vatankhah-Varnosfaderani, M.; Keith, A. N.; Cong, Y.; Liang, H.; Dobrynin, A. V.; Sheiko, S. S.; et al. Strained Bottlebrushes in Super-Soft Physical Networks. ACS Macro Lett. 2019, 8 (5), 530-534. 